\title{
LA COINVESTIGACIÓN EN COMUNIDADES ZAPOTECAS DE OAXACA. REFLEXIONES HACIA UN DIÁLOGO INTERCULTURAL
}

\section{The Co-inVestigation in OAXaca's Zapotecas COMMUNITIES.REFLECTIONS TOWARDS} AN INTERCULTURAL DIALOGUE

\section{Mario Enrique Fuente Carrasco \\ fuente@unsij.edu.mx}

Currículo: doctor en Ciencias Ambientales. Profesor-investigador de la Universidad de la Sierra Juárez. Sus líneas de investigación versan sobre economía ecológica; sustentabilidad rural y economía solidaria: conflictos ambientales distributivos y lenguajes de valoración de la naturaleza; aproximaciones epistémicas y praxis sociales vinculadas con la construcción de nichos de sustentabilidad: interculturalidad, agua, forestal, ecoturismo, ruralidad, educación y políticas públicas.

\section{David Barkin \\ barkin@correo.xoc.uam.mx}

Currículo: doctor en Economía. Profesor distinguido de la Universidad Autónoma Metropolitana e investigador emérito del Sistema Nacional de Investigadores. Sus líneas de investigación abordan estrategias alternativas para el manejo sustentable de recursos regionales; economía ecológica, desarrollo y economía solidaria.

\section{Ana Lilia Esquivel Ayala}

anali_1@hotmail.com

Currículo: maestra en Ciencias Económicas. Profesora-investigadora de la Universidad Autónoma Metropolitana. Sus líneas de investigación abordan la economía ecológica.

\section{Mario Fernando Ramos Morales yavasia@unsij.edu.mx}

Currículo: licenciado en Ciencias Políticas. Profesor-investigador de la Universidad de la Sierra Juárez. Sus líneas de investigación abordan la filosofía comunal de vida, mito, conocimiento, ética y medio ambiente (local-comunitario).

Recibido: 31 de agosto de 2017. Aceptado para su publicación: 30 de noviembre de 2017. Recuperado de: https://sinectica.iteso.mx/index.php/SINECTICA/article/view/793 DOI: 10.31391/S2007-7033(2018)0050-008

\section{Resumen}

El artículo es una autorreflexión metodológica de educación no formal basada en un proyecto colaborativo de investigación (coinvestigación) entre actores sociales y académicos (docentes y estudiantes). Los actores sociales son de las comunidades zapotecas de la Sierra Juárez de Oaxaca y los actores académicos pertenecen a dos universidades públicas. Exploramos la reflexión desde cuatro dimensiones: la posibilidad de participar en un diálogo intercultural; el alcance de la propuesta en tiempo y espacio; las características operativas de la coinvestigación; y una autoevaluación de los investigadores comunitarios, que se enfoca a su contribución en esta actividad intercultural. Palabras clave: metodología, investigador comunitario, diálogo intercultural, coinvestigación. 


\begin{abstract}
The article is a methodological self-reflection of non-formal education based on a collaborative research project (co-investigation) between social and academic actors (teachers and students). The social actors are from the Zapotec communities of the Sierra Juárez of Oaxaca and the academics are from two public universities. Four dimensions reflection are explored: The (abstract) possibility of participating in an intercultural dialogue; the scope of the proposal in time and space; its operational characteristics of this co-research; and a self-evaluation of the community researchers, focusing on their contribution to this intercultural activity.
\end{abstract}

Keywords: methodology, community researcher, intercultural dialogue, coresearch.

\title{
ANTECEDENTES
}

rente al enfoque convencional de investigación de las universidades de ubicar a los actores sociales como objeto de estudio, un conjunto de profesores de la Universidad de la Sierra Juárez (UNSIJ) y de la Universidad Autónoma Metropolitana-Xochimilco nos dimos a la tarea de explorar otros enfoques con un carácter más intercultural. El documento expone parte de esos intentos y reflexiones. Estas acciones se ubican dentro de uno de los ámbitos de la educación intercultural expresada en una de las funciones sustantivas de las universidades: la investigación.

Existen diversas aportaciones en estas tareas, las cuales son altamente divergentes. Van desde aproximaciones como las de Brembeck y Thompson (1973) y Bartolomé (2003) hasta posturas como las de Restrepo (2003). Las más cercanas a este enfoque son las visiones de Dietz y Materos (2011) y Dietz (2011), las cuales coinciden con este trabajo en explorar un diálogo en la dimensión "interactoral" como una tarea de tipo etnográfico; con ello surge la posibilidad de establecer un diálogo entre grupos culturalmente heterogéneos no asentado desde la educación formal, sino desde el ámbito no formal de la reflexión-acción (investigación). En nuestro caso se enfoca a un diálogo entre los habitantes de comunidades con ascendencia de la cultura mesoamericana frente al espectro de académicos (profesores y estudiantes).

De manera paralela, Barkin y Lemus (2015) hacen un análisis de la importancia de los trabajos de coinvestigación para nutrir la agenda de una gobernanza ambiental con justicia ambiental. Su propuesta está basada en una importante revisión de la literatura emergente en los ámbitos metodológicos y epistemológicos diferentes, así como la aglutinación de ciertos principios derivados de un conjunto de diversas experiencias comunitarias.

Los trabajos iniciados por los investigadores de manera colaborativa con las comunidades fueron intensificados en 2011 y consistieron, básicamente, en la realización de "Encuentros serranos" anuales dados en el espacio de la UNSIJ y, en los primeros, con la participación de la Fundación Comunalidad. En estos encuentros se invitaba a las comunidades para que expusieran a los alumnos de la UNSIJ una serie de reflexiones y acciones que ellos discutían y ponían en práctica para percibir, analizar y enfrentar soluciones a diversos problemas socioambientales. Se trataba de que los alumnos (y académicos) fueran sensibles e identificaran, reconocieran y valoraran la relevancia de mantener un diálogo con las comunidades para una mayor comprensión del potencial del saber comunitario frente a enfoques tecnocráticos y unilaterales que prevalecen desde la academia y las 
dependencias gubernamentales. Los primeros tres encuentros versaron sobre los recursos naturales, el agua y el ecoturismo.

Con la incursión en el proyecto de la UAM-X en 2013 surgieron no solo nuevos niveles y retos de interacción, sino un conjunto de términos diferentes a los analizados en los encuentros serranos. En los aspectos operativos se formalizó la invitación y participación de representantes de las comunidades. La invitación formulada por la UNSIJ y la UAM-X se dirigió a las autoridades correspondientes para que estas designaran a un representante que pudiera participar de una manera más constante en el proyecto. Un aspecto relevante es que se solicitaba que dicho representante tuviera (o hubiera tenido) una relación con los cargos designados por las propias comunidades. Con ello se pretendía que el participante estuviera involucrado de modo directo en las dinámicas internas de las comunidades.

Este trabajo colaborativo tuvo un salto cualitativo en 2014 cuando UNITIERRAOaxaca realizó un seminario en el que presentó una propuesta a nuestras actividades de investigación denominada reflexión en la acción. La nota de autorreflexión metodológica (todavía preliminar) del pretendido diálogo intercultural y de la noción de "investigador comunitario" se expone en este artículo.

Centrada en esta dinámica, pero aún desde el ámbito institucional, la actividad se registró como un proyecto de investigación convencional con el título "Soluciones locales para una justicia ambiental", que tenía la particularidad de colocar como eje metodológico el trabajo colaborativo con diversos actores sociales. Las actividades se realizaron con las comunidades zapotecas ubicadas en la Sierra Juárez de Oaxaca (SJO); entre ellas, Santa Catarina Lachatao, San Pedro Nexicho, Santa María Yavesía, Tanetze de Zaragoza, San Baltazar Yatzachi y Santa María Yahuiche.

\section{OBjetivo}

Del amplio trabajo llevado a cabo en dicho proyecto, este documento se acota a uno de los objetivos: "Diseñar una propuesta metodológica de investigación que favorezca el carácter intercultural entre los actores sociales de comunidades de la Sierra Juárez de Oaxaca y académicos con el fin de visibilizar y revalorar los procesos desplegados por las comunidades desde lo local".

\section{Problemática}

La acotación del objetivo permitió centrar la problemática en las siguientes cuestiones: ¿ंes posible establecer un diálogo intercultural entre actores sociales y académicos en el marco de un proyecto de investigación?, ¿cómo desplegar una propuesta operativa de coinvestigación que permita favorecer un diálogo intercultural de/con los investigadores comunitarios con ascendencia en la cultura zapoteca de la región de la SJO frente a un grupo de estudiantes y profesores universitarios?

Las aproximaciones a estas cuestiones derivan, a su vez, de otras preguntasproblema más específicas centradas en la figura del actor social como parte activa en la investigación sobre la realidad comunitaria; entre ellas, las siguientes: ¿cuáles son los rasgos esenciales para hablar de diferencias culturales entre grupos humanos?, ¿entre los sujetos identificados como académicos existen, a su vez, diferencias culturales?, y ¿los grupos participantes (académicos-actores sociales) asumen una "identidad" cultural diferente entre sí?

En caso de respuesta afirmativa, entonces ¿̇en qué grado?, ¿existen posibilidades de entablar una relación intercultural entre estos participantes?, ¿cuál es el 
objetivo de tal interrelación?, ¿̇en esta interrelación existe una imposición sutil, resistencias o apropiaciones?, ¿ existe una contribución para las partes por participar en esta interrelación?, ¿la visión de la academia es la que permitirá definir cuáles saberes son pertinentes de incorporar en esta investigación?, ¿es una opción metodológica, en estas tareas, el diálogo intercultural?, y ¿cuáles son los aspectos que habrá que tomar en cuenta para vigilar un adecuado diálogo intercultural, sobre todo su traslado a un tema de "investigación" sugerido desde una de las partes (de la academia)?

Debemos señalar que la aproximación a estas interrogantes es altamente compleja, ya que involucra una serie de manifestaciones amalgamadas a una compleja noción de cosmovisión de los participantes con la ascendencia en la cultura zapoteca frente a otras visiones (entre ellas, la de los académicos). Este atributo (cosmovisión) presenta peculiaridades con alto grado de dificultad para expresarse en forma empíricamente (Broda y Báez, 2001).

Con base en la problemática, partimos de cinco premisas que destacan la importancia de fomentar la coinvestigación de tipo intercultural:

- La asunción del modelo neoliberal por parte del Estado mexicano está instrumentando una forma de gobernación (gobernanza) caracterizada por evadir su responsabilidad (social y ambiental) y, al mismo tiempo, delegar este compromiso hacia otros actores no gubernamentales, de manera específica a un grupo cultural dominante económicamente. Dicho grupo impone su visión de sociedad, de mundo, de ciencia y del concepto mismo de investigación. Se trata de un modelo que asocia la idea de desarrollo con el paradigma de un crecimiento económico y su concentración, pero sin tomar en cuenta las consecuencias socioambientales (la justicia ambiental). En este entorno universalista es importante visibilizar otras narrativas ocultas, incluso por la academia.

-En coincidencia con diversos autores (Barkin y Fuente, 2013; Hogenboom Baud y Castro, 2012; Toledo y Barrera, 2008; Toledo, 2015; Vergara-Campus, 2011; Santos y Rodríguez, 2011), reconocemos el potencial geopolítico y socioambiental de los habitantes rurales del continente latinoamericano (en especial de las regiones rurales) en la búsqueda de salidas a los temas emergentes sobre la crisis ambiental global (calentamiento global, biodiversidad, vulnerabilidad hídrica) y alternativas a los problemas de desigualdad social, exclusión e injusticia.

- La relevancia de las aportaciones de algunas praxis comunitarias expresadas en el ámbito local tiene implicaciones mucho más amplias de lo delimitado espacialmente como lo "local". Barkin y Fuente (2013) señalan que el desarrollo de capacidades autogestionarias en cada comunidad se fortalece y tiene como espacio de construcción de autonomías el nivel de alianzas intracomunitarias.

- La relevancia de las expresiones culturales locales y regionales de la SJO destaca su cosmovisión, capacidades organizativas productivas y políticas de las comunidades. En esta sobresale la palabra comunalidad 
frente a la académica de gobernanza. La primera es más familiar en el lenguaje cotidiano del grupo a partir de las discusiones realizadas por intelectuales encarnados (Díaz, 2007; Martínez-Luna, 2003, 2010, 2013), en organizaciones como la Fundación Comunalidad y otros actores académicos que han desarrollado actividades en esta región. Señalamos la idea de intelectual encarnado para diferenciarlo de las connotaciones del intelectual orgánico de Gramsci; es decir, las reflexiones y funciones organizativas que cumple el intelectual frente a las ideas dominantes no se efectúan en una esfera externa (el partido, el sindicato), sino que estas son aprendidas en su pertenencia en la comunidad. En contraparte a la comunalidad, la noción de gobernanza se ha usado de manera excesiva en el lenguaje gubernamental y de diversas organizaciones no gubernamentales internacionales (como el Banco Mundial, el Programa de las Naciones Unidas para el Desarrollo, entre otras). Debemos destacar que la palabra comunalidad permite identificar (dentro del discurso de Bonfil [1982] de lo propio y lo ajeno) una serie de elementos culturales frente a otros dominantes: lo comunal-la propiedad privada; el interés comunitario-el individual; el uso de la democracia deliberativa (asamblea)-la democracia representativa (por partidos políticos), entre otras (Hernández-Díaz, 2007).

- Las praxis comunitarias narradas en forma directa por los actores sociales locales no son estáticas ni homogéneas. Reconocemos que estas cotidianamente mantienen contradicciones en la construcción de espacios autogestionarios, los cuales presentan fortalezas, pero también diversos niveles de fragilidad. Son espacios que demandan una constante reconfiguración en función de las señales de las instituciones de la racionalidad económica de corte neoliberal, tanto del Estado como del mercado. Por ello, su configuración es dinámica e implica, al mismo tiempo, el enfrentamiento de diversas luchas políticas no solo hacia el exterior, sino al interior de las mismas comunidades.

\section{Metodología. DesarRollo de la propuesta}

Uno de los supuestos de la estrategia de reflexión en la acción parte es la posibilidad de integrar la palabra de las comunidades a través de actores sociales locales con la figura de "investigadores comunitarios". Ello fue reconocido en el seminario y en la experiencia de UNITIERRA, especialmente en las reflexiones con Gustavo Esteva de manera directa y las plasmadas en algunos de sus textos $(2004,2014)$. A continuación hacemos una aproximación a esta exploración de rutas hacia una metodología desde los principios de un diálogo intercultural.

\section{Una reflexión inicial: la cultura y el universalismo cultural}

Hay matices culturales que permiten identificar diferencias culturales entre los académicos y los actores sociales participantes. Tal afirmación lleva en forma implícita una noción específica de cultura, la cual se tendrá que desenmarañar. De acuerdo con Esteva (2004), esta noción puede incluir componentes mutuamente articulados de diferente nivel de visibilidad (tangibles) y profundidad. Los que por tradición se identifican como rasgos culturales son los "más" visibles y, por ello, los más externos. 
En este cajón (externo) se ubican, por ejemplo, las expresiones como la lengua, la comida, el arte y, en general, las costumbres cotidianas y comportamientos de un grupo. Sin embargo, también existe otro grupo de rasgos estructurales no tan fácilmente perceptibles por un miembro fuera del grupo, pues requieren una cuidadosa y prolongada observación y análisis. Dentro de este rubro están presentes los aspectos organizacionales y las prácticas sociales, como las expresadas desde la estructura familiar y las normas jurídicas y políticas. Por último, se tiene otro tipo de rasgos más profundos que son clave en la determinación de los citados aspectos estructurales (o morfológicos): "Es el nivel del mito, de la cosmovisión, de la fuente del sentido, del horizonte de inteligibilidad, de lo que a veces llamamos espiritualidad o raíz del conocimiento" (Esteva, 2004, p. 77).

En su intento de precisión de tal palabra (cultura), Esteva trasciende otro nivel de precisión a partir de lo formulado por Panikkar (1993), que consideramos relevante para responder a las interrogantes formuladas; sin embargo, también pueden incorporarse para analizar otros asuntos como la educación intercultural y los derechos humanos, sobre todo en comunidades originarias:

Cultura es el mito englobante de una sociedad o un grupo en un momento dado del tiempo y del espacio. Mito sería, en esta noción, el horizonte de inteligibilidad en el que todas nuestras percepciones de la realidad adquieren sentido. El mito nos ofrece el marco en el cual se inscribe nuestra visión del mundo. Es lo que permite y condiciona cualquiera de nuestras interpretaciones de la realidad. Permite ver, como la luz, pero habitualmente, como a ella, no podemos verlo (Esteva, 2004, p. 77).

Esta precisión tiene varias implicaciones: la primera es que la cultura (ajena) no es objetivable con los métodos ortodoxos de la investigación, sobre todo el dominante positivista y cuantitativo. Una segunda consideración abre una ventana hacia un tipo de interculturalidad: se refiere a un rechazo a identificar valores culturales universales desde los cuales se pueda formular un criterio supracultural y una jerarquización, tal como lo ha pretendido la cultura occidental capitalista. No obstante este rechazo, también se tiene precaución hacia un relativismo cultural extremo. En vez de ello, Esteva (2004) considera la posibilidad de una relatividad: “Cada cosmovisión, cada afirmación, cada noción, es relativa a su contexto. Nadie posee, desde ninguna cultura, una visión que las pueda abarcar a todas" (p. 78).

\section{La situación de la interculturalidad y el diálogo intercultural}

Hay un asunto relevante de considerar cuando miembros de dos grupos se relacionan culturalmente: se refiere al tipo de orientación que imprime uno de ellos sobre otro. Esto puede ser a través de una imposición o una persuasión, pero también tiene su reverso: la respuesta del otro grupo a esos procesos, ya sea de resistencia o de asimilación, absorción, apropiación. Una vertiente de esta interacción es el enriquecimiento cultural. Esteva (2004) se refiere a este proceso como "la tradición de cambiar la tradición de manera tradicional, lo que da continuidad histórica a una cultura y permite que su cambio, su dinamismo, no la disuelva" (p. 79). De manera semejante, Wolf (1987) destaca la misma observación en su estudio histórico.

Frente a lo anterior, es necesario matizar el tipo de situación de interculturalidad que se aspira en el trabajo como una dinámica de quien adquiere la conciencia de 
que existen otras personas, valores y culturas, y reconoce que no es posible el aislamiento ni tampoco renunciar a su propia cultura. Esa conciencia admite la limitación de toda cultura, la relativización de todo lo humano, y en vez de refugiarse en la propia, de intentar aislarse, alejándose del otro o suprimiéndolo, se anima a interactuar con él desde el reconocimiento de su otredad.

A pesar de que se plantea que no existe un lenguaje intercultural, hay un contexto que trata de imponer un pensamiento único (universal) con un sentido monocultural. Ante ello, Esteva (2004) llama a poner a prueba la opción del diálogo intercultural, que ha de ser un diálogo dialogal:

Es un diálogo que trasciende los planos del logos de cada una de las culturas que entran en relación, sus sistemas conceptuales, sus razones y valores. Implica una mutua apertura a la preocupación del otro, para compartir alguna guía, sospecha, inspiración, ideal, o cualquier elemento que ambas partes puedan compartir y ninguna de ellas controle (p. 79).

En esta disposición hay otro componente relevante enfáticamente señalado por Esteva (2016): la clave de este diálogo intercultural se encuentra en el escuchar. El diálogo no es posible si los dialogantes no se escuchan mutuamente. En coincidencia con Lenkensdorf (2008), se indica lo siguiente: para acercarse a la otredad del otro hace falta imaginar mucho más que una cosmovisión, en que se hace predominar el sentido de la vista, en una clara tradición occidental. Se necesita concebir una cosmoaudición, y de hecho una cosmovivencia. De esta forma, entonces, el escuchar, trascendiendo el logos, conduce al hacer, y es ahí, en la práctica, en donde finalmente puede tener lugar el diálogo intercultural.

Este asunto del diálogo intercultural también es complejo en la trama de vincular una interacción entre académicos y actores sociales, como se pretende en este proyecto. Al respecto, se identifica que cada participante utiliza de manera diferente el lenguaje: mientras que los académicos usan términos, conceptos o categorías, los actores sociales que participan en el proyecto emplean las palabras para dar cuenta de su realidad. Provoca problemas porque la ciencia extrae estas palabras para traducirlas a sus términos, y con ellas acotar una explicación de la realidad.

Ubicados en esta aparente disyuntiva (términos-palabras), Esteva indica que con los términos que usa la ciencia construimos un mundo al que atribuimos carácter "objetivo". Esta operación puede cumplir funciones útiles, pero hay quienes consideran ese mundo "objetivo", reducido a signos, como el único y más importante mundo real, como la única realidad precisa y cognoscible. La perspectiva científica se sobrepone a la perspicacia humana. La confusión entre términos y palabras es el principio de la decadencia de una cultura. Quiere decir que ya no está viva: que se ha fosilizado. El lenguaje ha dejado de ser mediador y se vuelve intermediario. El mediador está en la naturaleza del hablante y del oyente; no simplemente los conecta.

Hay otros componentes que Esteva sugiere poner especial atención y que son difíciles de escuchar como términos. Uno de ellos es el de los bienes comunes y el otro el uso de nosotros. La observación es muy pertinente en este intento de un diálogo intercultural con las praxis de las comunidades de la SJO, y con el tema de la gobernanza. Como ejemplo de esta dificultad está el desarrollo teórico formulado por Ostrom en su clásico libro Gobierno de los comunes (2000). Su propuesta es 
una contribución frente al esquema dogmático positivista deductivo de la teoría económica neoclásica para descalificar formas de gobierno fuera de los axiomas del mercado. Sin embargo, sus planteamientos siguen anclados aun en las premisas de la cultura universal occidental que concibe la sociedad como conjunto de individuos y presenta una versión más sofisticada del individualismo metodológico (actor racional ligado a intereses de costo-beneficio).

\section{Resultados. CREACIÓn de LA FIGURA de INVESTIGAdOR COMUNITARIO}

La incursión de los actores sociales en el seminario de UNITIERRA-Oaxaca detonó un cambio cualitativo en la concepción de la participación de los actores sociales en la investigación a través de la figura del "investigador comunitario". Lo descrito a continuación no tiene una intención anecdótica; quiere apuntalar cómo el grupo ha asumido tal figura y cómo se ha separado de ella. Esto tiene implicaciones no solo metodológicas, sino éticas en los trabajos de coinvestigación diseñados desde espacios externos a las comunidades.

Los actores sociales participaron en noviembre de 2012 en un encuentro con fuerte acento académico. Asistieron a una de las reuniones de investigadores universitarios del proyecto internacional Gobernanza Ambiental en América Latina y el Caribe, celebrado en Morelia. A partir de este se institucionalizó un seminario permanente para favorecer una mayor comprensión de los términos usados en el encuentro de Morelia y también para ir definiendo una estrategia de mejor comunicación o diálogo entre la academia y los representantes comunitarios. De esa forma, y con la integración de los actores sociales y académicos al proyecto de la UAM-X, se logró mayor participación en diversos foros externos, como los dos congresos internacionales celebrados en 2013 en Oaxaca y uno en 2014 en Xalapa, Veracruz.

En el seminario de UNITIERRA-Oaxaca se interactúa no solo con otros académicos, sino, y sobre todo, con otros actores sociales no adscritos a instituciones formales de educación o investigación. Se trata de compañeros investigadores comunitarios de Valles Centrales. Esto representó un cambio fundamental en el concepto de investigador y también en el lenguaje (palabras-términos) de reflexión de la acción. Las principales consecuencias identificadas son dos:

- El nivel de interacción y uso de palabras exigieron una mayor atención para escuchar.

- Los investigadores de las comunidades de la SJO percibieron la relevancia de incorporar a otros miembros de su comunidad que no habían estado familiarizados con la dinámica comunitaria local, pero sí con el conjunto de código de intercambio de un "seminario".

La respuesta ante tal demanda fue la incorporación de una nueva figura: un investigador comunitario estudiantil. Los resultados de esta interacción se están evaluando, pero sin duda ha venido a enriquecer la propuesta metodológica asumida en un principio.

De manera esquemática, el conjunto de las complejas relaciones de la propuesta se representa en la figura. Entre las vinculaciones más relevantes de los coinvestigadores comunitarios se destacan los siguientes cinco niveles diferenciados: 
f1: Relación del coinvestigador con las autoridades. En este caso, la figura reconocida al interior de la comunidad es como ciudadano o comunero.

f2: Son las relaciones que mantiene con las universidades dentro del proyecto, tanto con los profesores como con estudiantes de dichas universidades. Se trata de vínculos más identificados como investigadores.

f3: Se presenta una relación con entidades de difusión, como los citados congresos académicos o de intercambio de experiencias.

f4: Son los vínculos de intercambio de experiencias fundamentalmente en el seminario mantenido con compañeros de UNITIERRA, entre ellos, con otros investigadores $\mathrm{o}$ activistas.

f5: Son las relaciones que mantuvieron los coinvestigadores con estudiantes de su propia comunidad. No todas las comunidades tuvieron estos vínculos con estudiantes.

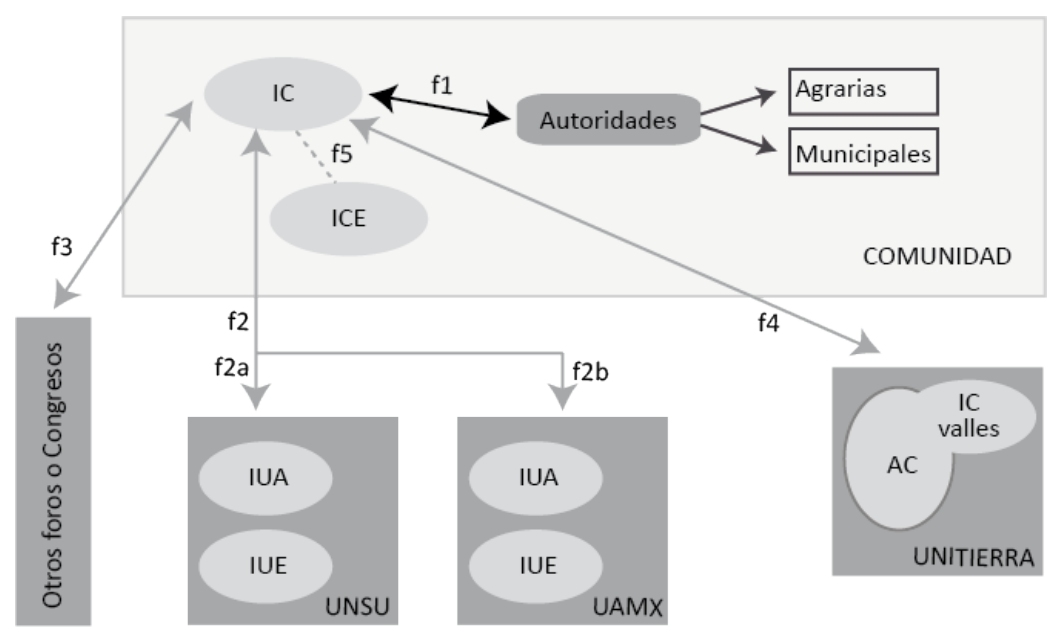

Figura. Tipo de interrelaciones del representante comunitario o del investigador comunitario. IC: Es ciudadano/comunero hacia el interior de la comunidad (f1), y se identifica en el proyecto como investigador comunitario hacia la esfera externa (f2-Universidades, f3-Foros como congresos y $f 4$ ante UNITIERRA).

ICE: investigador comunitario en su calidad de estudiante.

IUA: investigador universitario académico de la UNSIJ y la UAMX.

IUE: investigador universitario estudiante de la UNSIJ y la UAMX.

AC: alianza de colectivos de UNITIERRA.

IC Valles: investigadores comunitarios de Valles Centrales.

\section{DISCUSIONES}

\section{Acotaciones de la propuesta metodológica}

La tarea para desarrollar una propuesta metodológica basada en un diálogo intercultural entre investigadores académicos y comunitarios presentó diversas acotaciones relevantes. Enunciamos las principales: la primera indica que la aparente homogeneidad de la SJO no es tal. Es evidente que la zona geográficamente comparte similares antecedentes históricos, culturales y ambientales, pero también hay fuertes matices microrregionales y arreglos comunitarios. Con ello se pretende plasmar que las reflexiones emanadas del documento no necesariamente 
pueden extenderse a toda la región para explicar o comprender el trinomio citado ni tampoco como condiciones estáticas dentro de cada comunidad participante.

La segunda advertencia se refiere a la llamada "unidad de análisis" territorial, la cual es identificada como el cuarto nivel de gobierno: la localidad, pero a su vez integrada a un espacio territorial denominado comunidad agraria, cuyos principales lineamientos están previstos en el artículo 27 constitucional. En este sentido, las reflexiones de los investigadores comunitarios o sujetos sociales presentan una doble y complementaria identidad: la de ciudadanos (adscritos a la unidad administrativa municipal a nivel de localidad) y la de comunero (adscrito a la unidad productiva agraria).

En este punto es preciso hacer una acotación más detallada. La figura de "ciudadano" en las comunidades con ascendencia de la cultura zapoteca de la SJO se entiende de modo diferente al uso común en las zonas urbanas o en el lenguaje académico. La edad para ser ciudadano varía y está ligada a la posibilidad de cumplir ciertas obligaciones comunitarias antes que reclamar derechos. El estado civil, la edad, la condición de estudiante e incluso el género son factores que pueden estar relacionados con la condición de ciudadano. Otro punto relevante es que dicha categoría puede adquirir no solo un carácter individual, sino que puede ser representativo de la postura de la familia ante la comunidad o, de manera más específica, ante la asamblea o las autoridades. En este sentido, persisten dos tipos de asambleas y autoridades desde las cuales se construye la citada gobernanza local. Este punto tiene implicaciones metodológicas y éticas en la noción de "investigador comunitario" que se adquirió dentro del proyecto.

Hay una tercera consideración: la descripción de respuestas de cada comunidad no tiene inicialmente un carácter comparativo, sino integrador de una realidad compleja con diversas potencialidades y direccionalidades de sus niveles de comunalidad. Ello, por otra parte, no excluyó la realización de una serie de balances específicos en algunos rubros respecto al citado trinomio, sobre todo a partir de la interacción de experiencias entre los investigadores comunitarios. Lo anterior se puede evidenciar en los análisis específicos del trabajo mencionados en la introducción.

Finalmente, debemos referir que las comunidades colaboradoras en el proyecto presentan una característica especial dentro de la interacción emblemática de comunidades de la SJO: no han participado en forma activa (o se han retirado) del programa gubernamental asociado para consolidar un manejo forestal comunitario hacia su dimensión empresarial. Este último punto es relevante, ya que, en la actualidad, existe una fuerte identificación no solo por los académicos, sino por los políticos, por usar el aprovechamiento forestal como la identidad productiva de la SJO.

\section{Figura dual: investigador comunitario y ciudadano}

La reunión de Morelia catalizó la idea de rotular la figura del actor social como investigador. El grupo decidió explorar la noción de "investigadores comunitarios". En este contexto, los actores sociales participantes de las comunidades en el proyecto destacaron dos asuntos relevantes en la construcción metodológica hacia el diálogo intercultural:

-Antes que investigadores comunitarios, se identifican como ciudadanos de sus respectivas comunidades, y como serranos en adhesión a su identidad regional. 
- Ratifican que el proceso de aprendizaje significativo está determinado en gran medida por su participación e intervención directa en la toma de decisiones de la comunidad, y la asamblea es uno de los espacios emblemáticos.

Esta matización tiene diversas implicaciones. Una de ellas es identificar la figura de investigador comunitario únicamente como una entidad que pretende comunicar algo de su comunidad hacia una entidad externa y dentro del contexto del proyecto de la UAM-X, pero no como una figura de comunicación hacia el interior de su comunidad; es decir, a diferencia de otros grupos de investigadores comunitarios que se califican como tales hacia dentro de su comunidad, ellos consideran que esta figura desvirtuaría su condición de ciudadano y comunero. La otra implicación es que reconocen que el mayor proceso de aprendizaje para conocer a su comunidad no ha provenido del uso de términos desde la esfera académica, sino de su activa participación en los citados ámbitos de la comunidad. Además, reconocieron que ese aprendizaje no tiene un sentido utilitario o de tipo academicista, más bien como parte de lo que nombran una filosofía de vida comunal.

A continuación transcribimos esa reflexión sobre la pertinencia de adscripción como investigadores comunitarios:

Uno de los elementos de la filosofía comunal de vida, también llamado comunalidad, es la autoridad comunal, la cual fundamenta la organización social comunitaria y que es un proceso social que se define en el cumplimiento de las obligaciones que tenemos todos los miembros de la comunidad, ciudadanos, comuneros, jóvenes y ancianos y poco a poco las mujeres, de participar en la vida social, cultural y política de la comunidad, así como el de contribuir y decidir en el destino de la misma. Esta autoridad se desarrolla en las instancias de deliberación, toma de decisiones y ejecución de los acuerdos y tiene a la Asamblea General, en sus dos vertientes: tanto de ciudadanos si se trata del municipio o agencia municipal, y de comuneros si se trata de la comunidad agraria, como su máxima autoridad. Otras instancias relevantes que le sigue son el Consejo de Ancianos, el Consejo de caracterizados, en otros lugares también llamados Consejo Consultivo. Estas dos instancias conforman un espacio de consulta y solo en algunos casos, de decisión. La autoridad se alcanza o tiene su máxima expresión en los sistemas de cargos, ya sean de carácter agrario, municipal, comunitario, cultural, social o religioso.

Estas instancias, la asamblea general de ciudadanos o de comuneros, existen desde hace muchos años en nuestras comunidades, y es en este espacio institucional colectivo donde los ciudadanos(as) y comuneros(as), hemos aprendido a reflexionar y analizar constantemente, ya que por lo menos hay una asamblea cada mes para discutir los asuntos que atañen a nuestras comunidades y se toman las decisiones colectivas y consensadas [sic].

Queremos recalcar que nuestras comunidades toman decisiones constantemente, y por lo tanto tenemos que discutir y reflexionar para la mejor toma de decisiones colectivas, que pueden ser para desarrollar alguna actividad o impulsar una obra de infraestructura al interior de la comunidad o para responder a propuestas y proyectos que vienen de afuera ya sea por las instituciones del Estado, por ONGs o personajes públicos. Estas decisiones pueden tener resultados positivos o negativos para la comunidad, incluso el rechazo o aceptación a propuestas externas; 
sin embargo, es importante señalar que como la decisión tomada es colectiva aquí tenemos un principio ético comunitario, la responsabilidad es de todos los ciudadanos o comuneros porque estuvimos de acuerdo con la decisión tomada. Es decir, en nuestras comunidades hay una responsabilidad colectiva como resultado de la toma de decisiones colectivas.

Por lo tanto, como hay esta responsabilidad, hemos aprendido, a lo largo de los años, a pensar, reflexionar y analizar los puntos, temas, situaciones a las que se enfrenta la comunidad para hacerle frente. Esto es muy importante señalarlo porque todos los que vivimos en la comunidad somos responsables del destino de nuestro pueblo-comunidad y asumimos esa responsabilidad. Por lo tanto, el análisis, la reflexión, el pensar y la toma de decisiones colectiva es un ejercicio cotidiano y permanente en nuestras comunidades, que viene desde nuestros antepasados y que seguimos y lo seguiremos haciendo.

Uno de los problemas centrales de la vida occidental, de la democracia moderna es precisamente la ausencia de responsabilidad ya no digamos individual sino colectiva. Por lo tanto, como miembros de las comunidades participantes en la vertiente del proyecto de la UAM-X sobre el tema de justicia ambiental hemos estado trabajando desde, para y con nuestras comunidades, como ciudadanos. Así, lo único que estamos haciendo es escribir lo que vemos; es decir, que somos como los Tlacuilos pero contemporáneos y no somos investigadores en el sentido expresado en otros encuentros, porque no tenemos ni el estatus, ni la categoría ni los beneficios de tal etiqueta. Esta figura de investigadores es para entendernos con los de afuera, que es una figura de enlace entre nosotros y los académicos.

En nuestras comunidades somos simple y llanamente ciudadanos, que participamos de la vida colectiva y asumimos nuestro papel en ese sentido. La figura convencional de investigador comunitario es entendida como ajeno a la comunidad, y que llega pensando que tienen la respuesta, y no es así. En este sentido, el investigador comunitario viene a ser otra figura más para articular lo local con lo externo, pero no para el caso de nosotros. Nosotros lo que estamos haciendo en el proyecto es trasmitir, hacia afuera, hacia los otros, lo que estamos haciendo en nuestras comunidades; mejor dicho, lo que nuestras comunidades están haciendo, y no para que nuestra labor se someta a un proceso de evaluación exterior; sino simplemente de interés para comunicar nuestra palabra.

Lo que el seminario ha aportado a nuestras actividades es, por un lado, confirmar lo que nuestros abuelos, y ahora nosotros, hacemos en la comunidad, pero por el otro lado, también brindó herramientas para una mejor realización de nuestras actividades cotidianas, y en dado caso para sistematizar nuestra interpretación de lo que acontece al interior.

\section{CONCLUSIONES}

La propuesta del diálogo intercultural mostrada en la experiencia de los actores sociales y de los universitarios dentro del proyecto "Soluciones locales para una justicia ambiental" no está terminada, pero sí ha dado pauta a ofrecer una forma de interacción cualitativamente importante desde el punto de vista epistemológico, ético y metodológico.

La propuesta pone en el centro de la discusión teórica a uno en la práctica: el intento de la academia por descolonizar su aproximación académica al análisis de/ con las comunidades, es decir, evitar un universalismo cultural, sobre todo en los 
conceptos de gobernanza y la etiqueta que se ha usado para nombrar al actor social como "investigador comunitario", implica reconocer que se trata de una relación entre entidades culturales diferenciadas, cuyo centro de interacción encierra el reconocimiento de componentes culturales inconmensurables, pero no por ello se mantuvo una postura cultural relativista.

Ante este dilema preferimos una opción de relatividad. Fueron varias las claves en la propuesta; una de ellas fue colocar el tema de escuchar como un importante instrumento de diálogo intercultural. Otro componente fue el uso del nosotros como paradigma diferenciado del individualismo metodológico. La distinción entre el uso de términos de los académicos y el de las palabras en las comunidades fue importante para enmarcar el alcance de nuestras discusiones.

Es conveniente mencionar que los coinvestigadores identificados en este documento como investigadores comunitarios reconocen que la interacción con los académicos fue relevante, al menos en cuatro sentidos más:

- Enriqueció su forma de interpretar sus procesos locales y, con ello, su interacción con sus comunidades.

- Han dado un papel importante a la sistematización de tales experiencias como una forma (no única ni la más importante) de documentar sus luchas entre sus congéneres.

- Les ofreció una mayor interacción entre experiencias comunitarias de la región, extrarregional e incluso internacional.

- Les permitió ponderar y revalorar sus acciones locales para la justicia ambiental respecto a otras luchas dadas. Ello es relevante porque manifiestan que en esas luchas entonces "no están solos".

La estrategia metodológica del diálogo intercultural y de la acción-reflexión comentada durante el seminario ha evidenciado la importancia del cuidado del uso de determinados conceptos utilizados de manera sutil y natural en los programas de gobierno. Destaca, por ejemplo, el uso del concepto de "necesidades" y el de "pobreza". El primero, en general, ha sido definido por entidades externas que en el fondo están ofertando o "vendiendo" sus servicios/productos a partir de un "requerimiento" inventado desde modelos de desarrollo ajenos. El ejemplo de Yatzachi es emblemático: identificaron y rechazaron la "venta" de programas de alcantarillado que requiere grandes cantidades de agua en una comunidad con problemas de abasto. Construyeron alternativas. La edificación de costosas y grandes plantas de tratamiento de aguas residuales ha caído en este grupo.

Lo mismo sucede con la palabra "pobre", en la que se etiqueta y se asigna poca o nula capacidad de grupos. Frente a esta noción de "necesidades" y de "pobreza", las comunidades están haciendo un inventario de su potencial organizativo, infraestructura y capacidades desde sus propios ámbitos comunitarios, para de ahí construir alternativas de desarrollo impulsado desde fuera.

El punto detallado anteriormente parece insignificante, pero tiene un fuerte peso en el ejercicio intercultural. Al respecto, Esteva (2016) indica:

Las palabras son puertas y ventanas de nuestra percepción; de las palabras que usamos depende nuestra experiencia del mundo. Y es eso lo que está inmediatamente amenazado y afecta nuestra capacidad de hablarnos [...] y de saber lo 
que pasa. En lugar de ser una forma autónoma de relación, el lenguaje se está convirtiendo en un dispositivo de aislamiento y un instrumento de manipulación y control.

Por su parte, Ribeiro (2016) apunta que "justamente debido a la gravedad de la crisis ambiental, tenemos que evitar el epistemicidio ecológico en curso que reduce la óptica, elimina conocimientos y destruye alternativas".

Existen otros baluartes identificados por las comunidades en este ejercicio de diálogo intercultural; destacan las siguientes, que se están revalorando en forma interna:

- Fortalecimiento de sus capacidades para la planeación, toma de decisiones, ejecuciones y supervisión de manera autónoma. En este punto cabe resaltar dos asuntos: la elección de sus autoridades locales fuera del sistema de partidos políticos y el carácter de la democracia directa expresada en la asamblea y en sus órganos.

- Fortalecimiento de los vínculos de colaboración intracomunitaria y la no reducción de la actividad a un ámbito puramente individual o familiar. Este atributo es esencial: la asociatividad y la comunalidad.

- Mantenimiento del trabajo comunitario (tequio, los cargos) como el componente de fuerza física relevante.

-El potencial biocultural territorial, tanto el comunitario como el familiar. - La autosuficiencia alimentaria -sobre todo de maíz y frijol- es un componente heterogéneo, pero presente en las comunidades.

- La construcción de fondos económicos locales, como lo representan las cajas populares o de ahorro locales.

-El fortalecimiento de proyectos productivos que están generando excedentes a la comunidad y a las familias.

\section{REFERENCIAS BIBLIOGRÁFICAS}

Barkin, D. y Lemus, B. (2015). Soluciones locales para la justicia ambiental. En Barbara Hogenboom, Michiel Baud y Fabio de Castro (coords.). Gobernanza ambiental en América Latina (pp. 297-331). Buenos Aires: CLACSO.

Barkin, D. y Fuente, M. (2013). Community forest management: Can the green economy contribute to environmental justice? Natural Resources Forum, vol. 37, núm. 3, pp. 200-210.

Bartolomé, M. A. (2003). En defensa de la etnografía. El papel contemporáneo de la investigación intercultural. Revista de Antropología Social, núm. 12, pp. 199-222.

Bonfil B., G. (1982). Lo propio y lo ajeno: una aproximación al problema del control cultural. En A. Colombres (comp.). La cultura popular. México: Premiá Editora.

Brembeck, C. S. T. \& Thompson, T. J. (eds.) (1973). New strategies for educational development: The cross-cultural search for nonformal alternatives. Boston: Lexington Books.

Broda, J. y Báez-Jorge, F. (coords.) (2001). Cosmovisión, ritual e identidad de los pueblos indígenas de México. México: Conaculta/Fondo de Cultura Económica.

Díaz, F. (2007). Comunidad y comunalidad. En S. Robles y R. Cardoso (comps.). Floriberto Díaz. Comunalidad, energía viva del pensamiento (pp. 34-50). México: UNAM. 
Dietz, G. (2011). Hacia una etnografía doblemente reflexiva: una propuesta desde la antropología de la interculturalidad. AIBR. Revista de Antropología Iberoamericana, vol. 6, núm. 1, pp. 3-26. Recuperado de http://www.redalyc.org/ $\mathrm{html} / 623 / 62321332002 /$

Dietz, G. y Mateos, L. S. (2011). Interculturalidad y educación intercultural en México. Un análisis de los discursos nacionales en su impacto en los modelos educativos mexicanos. México: Secretaría de Educación Pública.

Esteva, G. (2016). Para sentipensar la comunalidad. Bajo el Volcán, vol. 15, núm. 23, pp. 170-186. Recuperado de http://www.redalyc.org/html/286/28643473010/

Esteva, G. (2004). Desafíos de la interculturalidad. En Diálogos en acción, primera etapa. Interculturalidad (pp. 77-84). México: DGCP/Conaculta.

Hernández-Díaz, J. (coord.) (2007). Ciudadanías diferenciadas en un estado multicultural: los usos y costumbre en Oaxaca. México: Siglo XXI/IISUABJO.

Hogenboom, B., Baud, M. y Castro, F. de (2012). Gobernanza ambiental en América Latina: hacia una agenda de investigación integradora. Revista del Centro Andino de Estudios Internacionales, núm. 12, pp. 57-71.

Lenkersdorf, C. (2008). Cosmovisiones. México: Universidad Nacional Autónoma de México.

Martínez Luna, J. (2013). Textos sobre el camino andado. Oaxaca: Campo, AC/CSEIIO/ CMPIO/Plan Piloto/CNEII/CEEESCI.

Martínez-Luna, J. (2010). Eso que llaman comunalidad. Oaxaca: Conaculta/Campo, AC/Fundación Harp Helú.

Martínez-Luna, J. (2003). Comunalidad y desarrollo. Oaxaca: Conaculta/Campo, AC.

Ostrom, E. (2000). El gobierno de los bienes comunes. La evolución de las instituciones de acción colectiva. México: UNAM/CRIM/Fondo de Cultura Económica.

Panikkar, R. (1980). Words and terms. Roma: Istituto di Studi Filosofci.

Restrepo G., B. (2003, mayo). Investigación formativa e investigación productiva de conocimiento en la universidad. Nómadas, núm. 18, pp. 195-202.

Ribeiro, S. (2016). La nueva medida de todas las cosas: el carbono. La Jornada, 20 de agosto. Recuperado de http://www.jornada.unam.mx/2016/08/20/opinion/025a1eco

Santos, B. y Rodríguez Garavito, C. (2011). Ensanchando el canon económico y buscando alternativas. En Otra producción es posible. México: Fondo de Cultura Económica.

Toledo, V. (2015). Ecocidio en México. La batalla final es por la vida. México, DF: Grijalbo.

Toledo, V. y Barrera Bassols, N. (2008). La memoria biocultural: la importancia ecológica de las sabidurías tradicionales. Barcelona: Icaria.

Vergara-Campus, L. (2011, julio-septiembre). Globalización, tierra, resistencia y autonomía: el EZLN y el MST. Revista Mexicana de Sociología, vol. 73, núm.3, pp. 387-414. Recuperado de http://www.scielo.org.mx/scielo.php?script=sci_arttext \&pid=S0188-25032011000300001

Wolf, E. R. (1987). Europa y la gente sin historia. México: Fondo de Cultura Económica. 